\title{
High frequency attenuation measurements of lipid encapsulated contrast agents
}

\author{
D.E. Goertz ${ }^{\text {a,b,*}}$, M.E. Frijlink ${ }^{\text {a }}$, M.M. Voormolen ${ }^{\text {a,b }}$, \\ N. de Jong ${ }^{\mathrm{a}, \mathrm{b}, \mathrm{c}}$, A.F.W. van der Steen ${ }^{\mathrm{a}, \mathrm{b}}$ \\ a Biomedical Engineering Department, Erasmus Medical Centre, Room EE2302, Dr. Molewaterplein 50, \\ 3015 GE Rotterdam, The Netherlands \\ ${ }^{\mathrm{b}}$ Interuniversity Cardiology Institute of the Netherlands, 3501 DG Utrecht, The Netherlands \\ ${ }^{\mathrm{c}}$ Physics of Fluids, University of Twente, 7500 AE Enschede, The Netherlands
}

Available online 30 June 2006

\begin{abstract}
A number of recent studies have indicated the potential of ultrasound contrast agent imaging at high ultrasound frequencies. However, the acoustic properties of microbubbles at frequencies above $10 \mathrm{MHz}$ remain poorly understood at present. In this study we characterize the high frequency attenuation properties of (1) BR14, (2) BR14 that has been mechanically filtered (1 and $2 \mu \mathrm{m}$ pore sizes) to exclude larger bubbles, and (3) the micron to submicron agent BG2423. A narrowband pulse-echo substitution method is employed with a series of four transducers covering the frequency range from 2 to $50 \mathrm{MHz}$. For BR14, attenuation decreases rapidly from 2 to $10 \mathrm{MHz}$ and then more gradually from 10 to $50 \mathrm{MHz}$. For $2 \mu \mathrm{m}$ filtration, the attenuation peaks between 10 and $15 \mathrm{MHz}$. For $1 \mu \mathrm{m}$ filtration, attenuation continues to rise until $50 \mathrm{MHz}$. The agent BG2423 exhibits a diffuse attenuation peak in the range of 15-25 $\mathrm{MHz}$ and remains high until $50 \mathrm{MHz}$. These results demonstrate a strong influence of bubble size on high frequency attenuation curves, with bubble diameters of 1-2 $\mu \mathrm{m}$ and below having more pronounced acoustic activity at frequencies above $10 \mathrm{MHz}$.
\end{abstract}

(c) 2006 Elsevier B.V. All rights reserved.

Keywords: Contrast agents; Harmonic; Subharmonic; Microbubbles; High frequencies; Intravascular ultrasound

\section{Introduction}

There is increasing interest in the use of microbubble contrast agents at frequencies in the $10-50 \mathrm{MHz}$ range, where applications are possible in ophthalmology, small animal imaging, and cardiology. Several studies have investigated their use in 'linear' imaging mode using ultrasound biomicroscopy [1] and intravascular ultrasound (IVUS) systems $[2,3]$. More recent work has shown that it is also feasible to perform subharmonic and second harmonic imaging at high frequencies $[4,5]$. In these studies, both

\footnotetext{
* Corresponding author. Address: Biomedical Engineering Department, Erasmus Medical Centre, Room EE2302, Dr. Molewaterplein 50, 3015 GE Rotterdam, The Netherlands. Tel.: +31 10408 8042; fax: +31 10408 9445.

E-mail address: d.goertz@erasmusmc.nl (D.E. Goertz).
}

conventional commercial agents and experimental liposomal preparations with micron to submicron mean diameters have been employed.

The acoustic properties of microbubbles at high frequencies are not well understood at present, particularly with regards to knowledge of resonant bubble sizes and nonlinear scattering mechanisms. A reasonable hypothesis is that smaller bubbles are more active at high frequencies: for example, the resonant diameter of a free bubble at $20 \mathrm{MHz}$ is $0.65 \mu \mathrm{m}$. The application of current models for lipid encapsulated microbubbles also predicts increased acoustic activity in bubbles of diameters $1-2 \mu \mathrm{m}$ and below for frequencies above $10 \mathrm{MHz}$ (e.g. [6]). However, these models are not validated at high frequencies and shell properties required as input parameters are unknown. From an experimental perspective, it has been shown that mechanical filtration of BR14 (Bracco Research, Geneva) 
using 1 and $2 \mu \mathrm{m}$ pore sizes improves nonlinear scattering at 20 and $30 \mathrm{MHz}$ transmit frequencies [6]. Further, the experimental micron to submicron lipid encapsulated agent BG2423 (Bracco Research, Geneva) has been shown to have substantial nonlinear activity (subharmonics and second harmonics) for transmit frequencies of $20-40 \mathrm{MHz}$ $[5,7]$.

While attenuation measurements have been widely employed at lower frequencies $(<10 \mathrm{MHz})$ to gain insight into bubble resonant frequencies and shell properties [810], very little work has been done at high ultrasound frequencies $(>10 \mathrm{MHz})$ [11]. In this study we investigate the attenuation properties of lipid encapsulated contrast agents in the frequency range from 2 to $50 \mathrm{MHz}$.

\section{Methods}

\subsection{Measurement system overview}

Attenuation measurements were made using the pulseecho substitution method, an approach described in detail elsewhere [8]. A series of four commercially available transducers (Panametrics Inc., Waltham, MA) was used to cover the range of $2-50 \mathrm{MHz}$ (Table 1). Agent was situated in a sample chamber located within the transducer beam,

Table 1

Transducer models (Panametrics), characteristics, and frequency range

\begin{tabular}{llllll}
\hline $\begin{array}{l}\text { Transducer } \\
\text { and model }\end{array}$ & $\begin{array}{l}\text { Focal } \\
\text { length } \\
(\mathrm{mm})\end{array}$ & $\begin{array}{l}\text { Aperture } \\
(\mathrm{mm})\end{array}$ & $\begin{array}{l}\text { Path } \\
\text { length in } \\
\text { chamber }\end{array}$ & $\begin{array}{l}\text { Nom. center } \\
\text { frequency } \\
(\mathrm{MHz})\end{array}$ & $\begin{array}{l}\text { Frequency } \\
\text { range }(\mathrm{MHz})\end{array}$ \\
\hline 1. V304 & 75 & 25 & 42 & 2.25 & $2-3.5$ \\
2. V311 & 75 & 13 & 42 & 10 & $3-14$ \\
3. V317 & 50 & 6 & 29 & 20 & $8-30$ \\
4. V377 & 51 & 6 & 29 & 30 & $25-50$ \\
\hline
\end{tabular}

which entered the chamber through a mylar window and was then reflected off a steel plate located at focus. Two sample chambers were used, each one having two transducers (Fig. 1).

An overview of the hardware configuration is shown in Fig. 2. An arbitrary waveform generator (AWG 520 Tektronix, Beaverton, OR) was used to create low amplitude pulses, which were amplified with an RF power amplifier (2100 L, ENI, Rochester, NY for transducers 1 and 2; LPI-10, ENI, Rochester, NY for transducers 3 and 4) and sent to one of four transducers. On receive, signals were amplified (AU-1189 or AU-1263, Miteq Inc., Hauppage, NY), bandpass filtered, and then digitized (DP310, Acqiris, Geneva). A sequence of narrowband pulses was employed (20 cycles up to $10 \mathrm{MHz} ; 40$ cycles for 10 $50 \mathrm{MHz}$ ) to sweep the bandwidth of each transducer individually. The inter-pulse spacing was $200 \mathrm{~ms}$, with sequences repeated at a rate of $8 \mathrm{~min}^{-1}$. The use of narrowband pulses permitted the use of low transmit pressure levels that were controlled across a wide frequency range, in order to avoid potential pressure dependent attenuation effects [12] and thereby to enable comparison with linearized bubble models. This approach also extended the effective frequency range of each individual transducer, in terms

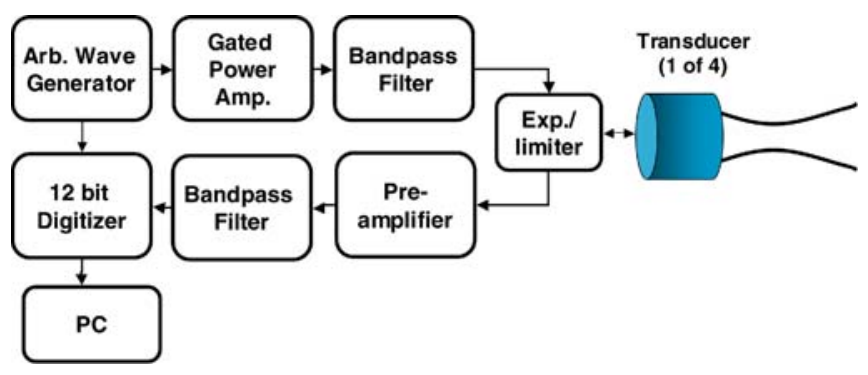

Fig. 2. Schematic overview of the hardware employed.

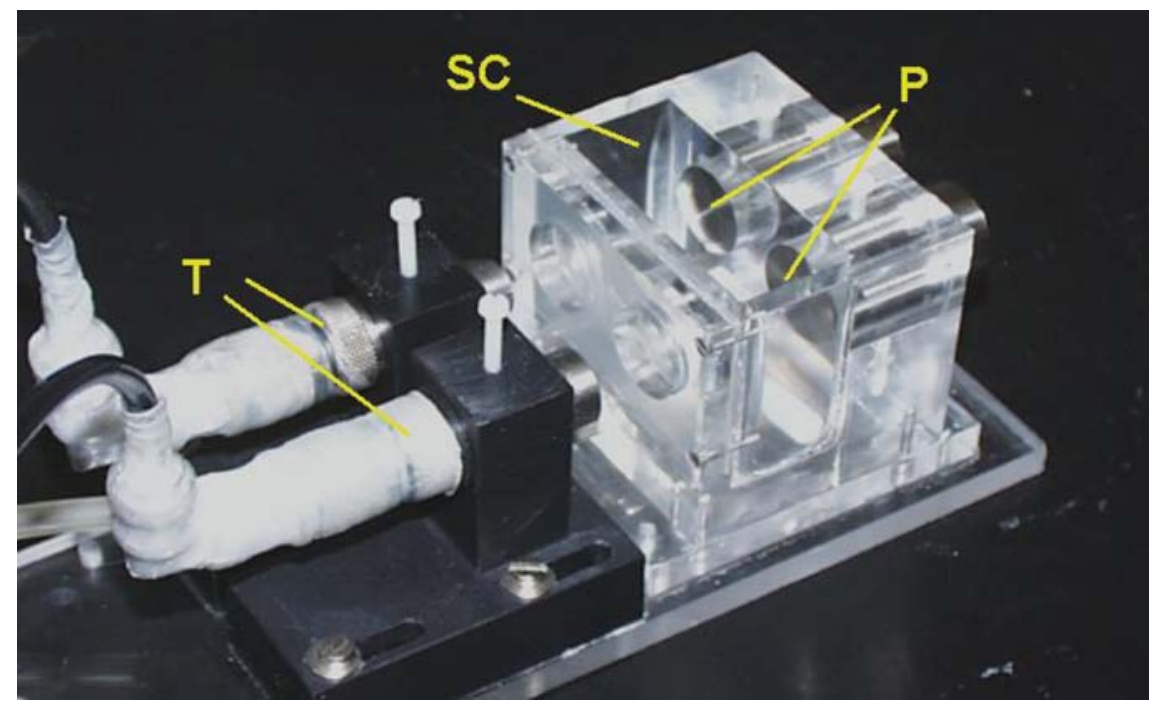

Fig. 1. Photograph of one of the sample chambers (SC) indicating the transducers $(\mathrm{T})$, and reflective steel plates $(\mathrm{P})$. These components were immersed in a water tank. 
of signal to noise ratio, relative to that which could be achieved with a wideband pulse. The pressure level used was $25 \mathrm{kPa}$, as measured with a $0.075 \mathrm{~mm}$ needle hydrophone (Precision Acoustics Ltd., Dorchester, UK) at focus in a water tank.

\subsection{Agent handling}

The agents examined were (1) BR14; (2) BR14 with its population modified by mechanical filtering ( 1 and $2 \mu \mathrm{m}$ pores sizes) to isolate small bubbles; and (3) BG2423, all experimental lipid encapsulated agents from Bracco Research (Geneva). According to size information provided by the manufacturer, BR14 has a mean volume weighted diameter of $6.8 \mu \mathrm{m}$ and $\mathrm{BG} 2423$ is comprised substantially of micron to submicron bubbles. Agent was diluted in Isotone ${ }^{\mathrm{TM}}$ to the ratios shown in Table 2. Filtration was performed by gravity feed of diluted agent through a porous polycarbonate membrane (Poretics 1 or $2 \mu \mathrm{m}$, GE Osmonics Inc), as also described in [6,8]. Measurements began 1 min after the agent was placed within the sample chamber, with agent undergoing gentle mixing

Table 2

Agent dilution ratios

\begin{tabular}{ll}
\hline Agent & Dilution ratio \\
\hline B14 & $1: 2000$ \\
BR14 (2 $\mu \mathrm{m}$ filter $)$ & $1: 300$ \\
BR14 $(1 \mu \mathrm{m}$ filter $)$ & $1: 150$ \\
BG2423 & $1: 700$ \\
\hline
\end{tabular}

with a magnetic stirrer. For a given sample, a total of eight measurements at each frequency were then performed during $1 \mathrm{~min}$. A total of four independent sample measurements were made for native BR14 and BG2423 (i.e. averaging 32 values per frequency in total), and two samples for filtered cases (i.e. averaging 16 values per frequency in total). All experiments were performed at room temperature.

\section{Results and discussion}

The attenuation results are shown in Fig. 3(a)-(d). For native BR14 (Fig. 3(a)), attenuation decreased steeply from 2 to $10 \mathrm{MHz}$ and more gradually from 10 to $50 \mathrm{MHz}$. These results indicate that its acoustic activity is most pronounced at lower diagnostic frequencies $(<10 \mathrm{MHz})$, as may be expected with a mean bubble size of $6.8 \mu \mathrm{m}$.

When BR14 is passed through a $2 \mu \mathrm{m}$ filter, the attenuation peak is shifted towards $10-15 \mathrm{MHz}$ before leveling off until $50 \mathrm{MHz}$ (Fig. 3(b)). This result suggests that there are bubbles below $2 \mu \mathrm{m}$ in diameter present within BR14 that have significant acoustic activity above $10 \mathrm{MHz}$. When BR14 is passed through a $1 \mu \mathrm{m}$ filter the attenuation at $10 \mathrm{MHz}$ is low, but continues to rise until $50 \mathrm{MHz}$ (Fig. 3(c)). This suggests that there are bubbles below $1 \mu \mathrm{m}$ in diameter present within BR14 that have significant acoustic activity up to $50 \mathrm{MHz}$. These data are consistent with the results of [6], which showed that high frequency (20 and $30 \mathrm{MHz}$ transmit) nonlinear scattering (subharmonic and second harmonic) from BR14 is improved with 1 and $2 \mu \mathrm{m}$ filtering. It should be noted however that while
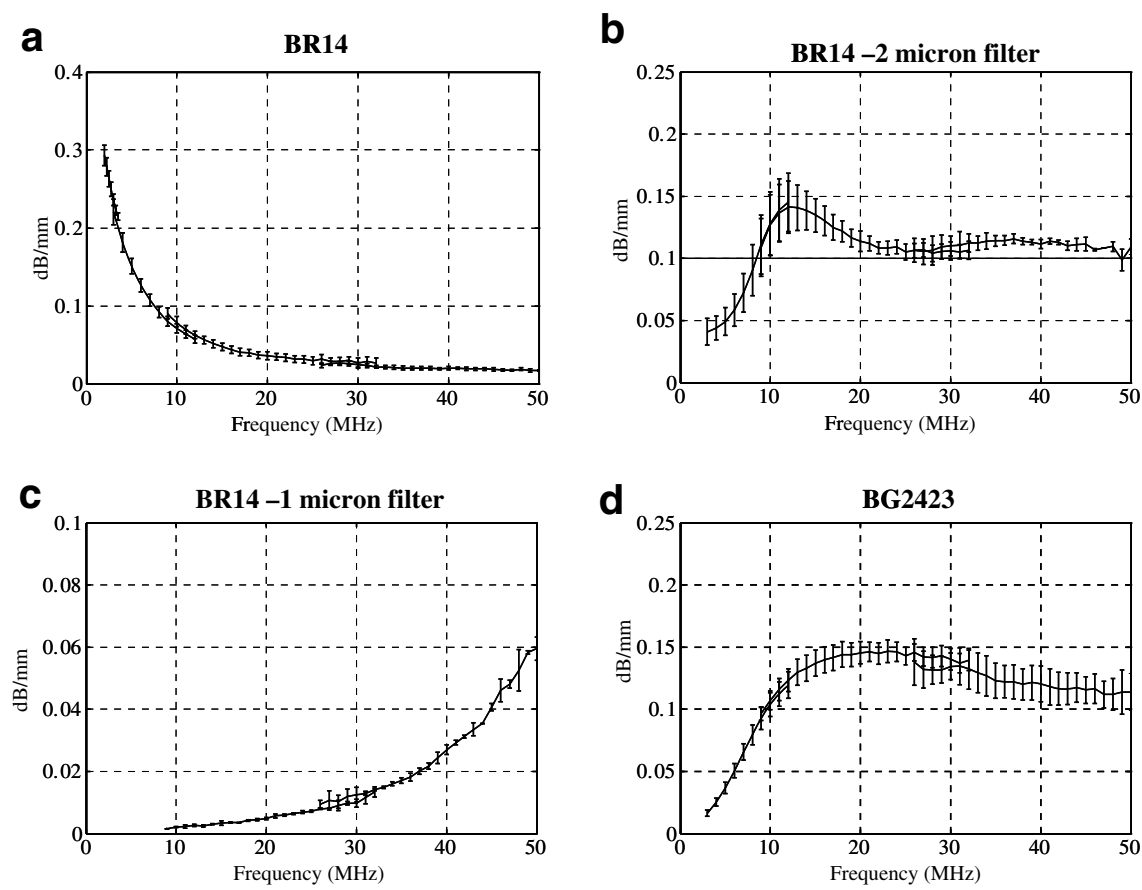

Fig. 3. Frequency dependent attenuation data (mean and standard deviations shown) for (a) BR14, (b) BR14 with $2 \mu \mathrm{m}$ filtering, (c) BR14 with $1 \mu \mathrm{m}$ filtering, and (d) BG2423. 
this particular approach to mechanical filtering was done under 'gentle' gravity fed conditions, it is not known if the filtration process may have modified the shell properties of the agent.

Attenuation for BG2423 exhibits a diffuse peak in the range of $15-25 \mathrm{MHz}$, and remains high until $50 \mathrm{MHz}$ (Fig. 3(d)). This agent has been found to have significant nonlinear activity at transmit frequencies from 20 to $40 \mathrm{MHz}[5,7]$. It is also notable that the form of the attenuation curve for this agent (comprised of micron to submicron sized bubbles) is intermediate between 1 and $2 \mu \mathrm{m}$ filtration results.

\section{Conclusions}

Frequency dependent attenuation has been measured in the $2-50 \mathrm{MHz}$ range for several lipid encapsulated bubble populations. The results of this study demonstrate a strong influence of bubble size on high frequency attenuation curves, with bubbles below 1-2 $\mu \mathrm{m}$ in diameter exhibiting more pronounced acoustic activity at frequencies above $10 \mathrm{MHz}$. This is consistent with the results of previous studies examining nonlinear activity of small bubble populations at high frequencies. These results also support the conclusion that agents with improved activity at high frequencies can be obtained through either the manufacture of small bubble agents or the modification of existing agents designed for use at lower frequencies. Future work with attenuation measurements will entail analyzing the data in combination with size distribution information to gain insight into shell properties and the relationship between bubble size and resonant frequencies above $10 \mathrm{MHz}$.

\section{Acknowledgements}

This work was financially supported by the Dutch technology foundation (STW). We thank Leo Bekkering for his contribution to the construction of the apparatus, and Bracco Research (Geneva) for providing the agent used in this study.

\section{References}

[1] C.X. Deng, F.L. Lizzi, R.H. Silverman, R. Ursea, D.J. Coleman, Imaging and spectrum analysis of contrast agents in the in vivo rabbit eye using very high frequency ultrasound, Ultrasound Med. Biol. 24 (1998) 383-394.

[2] C. Cachard, G. Finet, A. Bouakaz, A. Tabib, D. Francon, G. Gimenez, Ultrasound contrast agents in intravascular echography: An in vitro study, Ultrasound Med. Biol. 23 (1997) 705-717.

[3] S.M. Demos, H. Alkan-Onyuksel, B.J. Kane, K. Ramani, A. Nagaraj, R. Greene, M. Klegerman, D.D. McPherson, In vivo targeting of acoustically reflective liposomes for intravascular and transvascular ultrasonic enhancement, J. Am. Coll. Cardiol 33 (1999) 867-875.

[4] D.E. Goertz, E. Cherin, A. Needles, R. Karshafian, A. Duckett, P.N. Burns, F.S. Foster, High frequency nonlinear b-scan imaging of microbubble contrast agents, Trans. IEEE UFFC 52 (2005) 65-79.

[5] D.E. Goertz, M.E. Frijlink, N. de Jong, A.F.W. van der Steen, Nonlinear intravascular contrast ultrasound, Ultrasound Med. Biol. 32 (2006) 491-502.

[6] D.E. Goertz, M.E. Frijlink, A. Bouakaz, C.T. Chin, N. de Jong, A.F.W. van der Steen, The Effect of bubble size on nonlinear scattering from microbubbles at high frequencies, Proc. IEEE UFFC (2003) 1503-1507.

[7] D.E. Goertz, M.E. Frijlink, N. de Jong, A.F.W. van der Steen, Nonlinear scattering from a micron to submicron lipid encapsulated contrast agent, Ultrasound Med. Biol. 32 (2006) 569-577.

[8] N. de Jong, L. Hoff, T. Skotland, N. Bom, Absorption and scatter from microspheres: Theoretical considerations and some measurements, Ultrasonics 30 (1992) 95-103.

[9] N. de Jong, L. Hoff, Ultrasound scattering properties of Albunex, Ultrasonics 31 (1993) 175-181.

[10] L. Hoff. Acoustic characterization of contrast agents for medical ultrasound imaging. PhD thesis, Norwegian University of Science and Technology, 2000 Trondheim, Norway.

[11] C.M. Moran, R.J. Watson, K.A.A. Fox, W.M. McDicken, In vitro acoustic characterization of ultrasonic contrast agents at $30 \mathrm{MHz}$, Ultrasound Med. Biol. 28 (2002) 785-791.

[12] Q. Chen, J. Zagzebski, T. Wilson, T. Stiles, Pressure-dependent ultrasound attenuation measurements, Ultrasound Med. Biol. 28 (2002) 1041-1051. 